\title{
Magnetic Neutron Scattering Investigation and Kondo Effect in paramagnetic crystals
}

\author{
Azadeh Farzaneh $^{1 *}$, Mohammad Reza Abdi $^{1}$, Khadije Rezaee Ebrahim Saraee ${ }^{2}$ \\ ${ }^{1}$ Department of Physics, Faculty of Science, University of Isfahan, Isfahan, 81747-73441, Iran \\ ${ }^{2}$ Department of Nuclear Engineering, Faculty of Modern Science and Technologies, university of Isfahan, Isfahan, 81746-73441, Iran \\ Received 1 October 2011, Revised 25 November 2011, Accepted 30 November 2011, Available online 5 December 2011
}

\begin{abstract}
Paramagnetic inelastic neutron scattering have been considered to investigate the crystal of rare earth of cerium. In rare earth crystals The hybridization of the $\mathrm{f}$ electrons with electrons in the conduction band gives rise to a variety of interesting phenomena. In particular, magnetic form factor can use to help in the understanding of the wave functions of localized electrons, and the energy scales of magnetic fluctuations and characteristic temperatures will be analysed in inelastic neutron scattering . At high temperature, the magnetic scattering can be described by two coherent broad peaks. At low temperatures, one narrow inelastic peak indicates dynamic magnetic correlation and paramagnetic effective moment in cerium ions. These results allow us to establish that in $\mathrm{Ce}^{+3}$ ions, at low $\mathrm{T}$, inelastic scattering is contribution of the Kondo effect.
\end{abstract}

| paramagnetic cross section | atomic form factor | kondo effect | inelastic neutron scattering |

\section{INTRODUCTION}

The Physics of rare earth such as cerium has become very attractive because the hybridization of the $f$ electrons with electrons in the conductions band gives rise to a variety of interesting phenomena [1]. The use of neutron scattering is a powerful technique to understand the effect of magnetic structure and behaviour of crystal in measuring cross-section [2]. This technique is also used to distinguish magnetic properties of an unknown material and can be used to investigate the behaviour of the cerium ions in Kondo system. The Kondo effect describes the scattering of conduction electrons in cerium due to localized electrons that play role of magnetic impurities. In the Kondo system, as temperature decreases, the exchange interactions between localized electrons and conductions electrons are strongly renormalized and the scattering of the conduction electrons by the localized electrons is enhanced [3]. In the case of rare earth like $\mathrm{Ce}^{3+}$, the magnetic interaction between the localized $4 \mathrm{f}$ electrons takes place through the spin of the conduction band so that itinerant electrons play an important role [4]. In these materials, the non-perturbative growth of the interaction leads to quasi electrons with masses up to thousands of times the free electron mass, i.e., the electrons are dramatically slowed by the interactions. In a number of instances they actually are superconductors.

Corresponding author at: Department of Physics, Faculty of Science,
In this study, the localized electron system is used to derive the temperature dependence of the paramagnetic scattering cross section of $\mathrm{Ce}^{3+}$ which gives more accurate information about crystal structure because electrons are bounded in a form of crystal. Using Kondo effect, the total magnetic cross section changes are discussed in terms of temperature, phonon wave vector and scattering vector. The paramagnetic scattering cross section is measured for scattering vector $(\kappa)$ up to $0.8 \mathrm{fm}^{-1}$ in temperatures up to 2.5 $\mathrm{T}_{\mathrm{c}}$.

\section{EXPERIMENTAL}

\subsection{Materials, method and instruments}

The theory of neutron scattering has been treated in several text books and articles $[4,5,6,7,8,9]$. We recall here some basic results. We consider scattering of neutrons by a sample consisting of condensed matter which undergoes a change from a state $\lambda$ to a state $\lambda^{\prime}$ while the state of the neutron changes from $(\mathrm{k}, \sigma)$ to $\left(\mathrm{k}^{\prime}, \sigma^{\prime}\right)$. Briefly, by summing over all final states of the sample $\lambda^{\prime}$ and final polarization states $\sigma^{\prime}$, average over all initial states $\lambda$ of the sample, which occur with probability $\mathrm{p}_{\lambda}$ and over all initial states of neutron, which occur with a probability $\mathrm{p}_{\sigma}$ to obtain 


$$
\begin{aligned}
& \left(\frac{d^{2} \sigma}{d \Omega d E}\right)_{\substack{k \rightarrow k^{\prime}\\
}} \\
& =\frac{1}{N} \frac{k^{\prime}}{k}\left(\frac{m}{2 \pi \hbar^{2}}\right)^{2} \sum_{\lambda \sigma} p_{\lambda} p_{\sigma} \sum_{\lambda^{\prime} \sigma^{\prime}}\left|\left\langle k^{\prime} \lambda^{\prime} \sigma^{\prime}|V| k \sigma \lambda\right\rangle\right|^{2} \delta(E \\
& \left.+E_{\lambda}-E_{\lambda^{\prime}}\right)
\end{aligned}
$$

Equation (1) is called master formula of neutron scattering which forms the basis for the interpretation of all experimental neutron scattering data. This is a very general which the interaction potential $\mathrm{V}$ between the sample and the neutron is

$$
V=\gamma \mu_{N}\left\{2 \mu_{B} \widehat{\boldsymbol{\sigma}} \cdot \operatorname{curl}\left(\left(\frac{\hat{s} \times \boldsymbol{R}}{|\boldsymbol{R}|^{3}}\right)-\frac{e}{2 m_{e} c}\left(\widehat{\boldsymbol{p}}_{\boldsymbol{e}} \cdot \frac{\hat{\boldsymbol{\sigma}} \times \boldsymbol{R}}{|\boldsymbol{R}|^{3}}+\frac{\hat{\boldsymbol{\sigma}} \times \boldsymbol{R}}{|\boldsymbol{R}|^{3}} \cdot \widehat{\boldsymbol{p}}_{\boldsymbol{e}}\right)\right\}\right.
$$

From the equation (1) and (2), the cross section for the scattering of neutrons through the interaction potential given in equation (4):

$$
\begin{aligned}
& \frac{d^{2} \sigma}{d \Omega d E}=r_{0} \frac{\kappa^{\prime}}{\kappa}\left(\frac{1}{2} g f(\kappa)\right)^{2} \sum_{\alpha \beta}\left(\delta_{\alpha \beta}-\hat{\kappa}_{\alpha} \hat{\kappa}_{\beta}\right) \sum_{\lambda, \lambda^{\prime}} p_{\lambda} \sum_{l, d} \sum_{l, d^{\prime}}\left\langle\lambda\left|e^{-i \kappa \cdot R_{l d}} \hat{s}_{l d}^{\alpha}\right| \lambda^{\prime}\right\rangle \\
& \left\langle\lambda^{\prime}\left|e^{i \kappa \cdot R_{l} d^{\prime}} \hat{s}_{l^{\prime} d^{\prime}}^{\beta}\right| \lambda\right\rangle \delta\left(\hbar \omega+E_{\lambda}-E_{\lambda^{\prime}}\right)
\end{aligned}
$$

In general a magnetic ion in a crystal possesses both spin and orbital angular momentum while some magnetic ions (e.g. $\mathrm{Ce}^{+3}$ ) that would have no orbital angular momentum because of quenching by the crystalline field and they have a finite orbital angular momentum. This arises because of the action of spin-orbit coupling which, by mixing excited states into the ground state, aligns some orbital moment parallel or antiparallel to the spin. Dipole approximation is used because of main radius of the wave function of the unpaired electrons is much less than $|\kappa|^{-1}$.

Therefore, by considering LS coupling and dipole approximation, atomic form factor is defined [10]:

$$
f(\kappa)=\frac{g_{s}}{g}\left\langle J_{0}\right\rangle+\frac{g_{l}}{g}\left(\left\langle J_{0}\right\rangle+\left\langle J_{2}\right\rangle\right)
$$

Where $\mathrm{J}_{0}$ and $\mathrm{J}_{2}$ are spherical Bessel function, and $\mathrm{g}_{\mathrm{s}}, \mathrm{g}_{\mathrm{l}}$ and $\mathrm{g}$ are the gyromagnetic ratios which for $\mathrm{Ce}^{+3}$ paramagnetic, $\mathrm{g}_{\mathrm{s}}=0.4, \mathrm{~g}_{\mathrm{l}}=0.8$ and $\mathrm{g}=1.2$ are considered [11].

To express (3) in terms of a correlation function we proceed writing the delta function in terms of an integral representation. Hence, the partial differential cross section gets

$$
\frac{d^{2} \sigma}{d \Omega d E}=r_{0}^{2} \frac{k^{\prime}}{k}\left(\frac{1}{2} g f(\kappa)\right)^{2} \sum_{\alpha \beta}\left(\delta_{\alpha \beta}-\hat{\kappa}_{\alpha} \hat{\kappa}_{\beta}\right) \frac{N}{2 \pi \hbar} \int_{-\infty}^{\infty} d t \int d r e^{i(\kappa, r-\omega t)} \Gamma_{\alpha \beta}(r, t)
$$

where $\mathrm{N}$ is the number of unite cells. The spin-dependent forces are small hence the movement of each ion is independent of spin orientation, therefore

$$
\Gamma(r, t)=\frac{1}{N} \sum_{l d} \sum_{l^{\prime} d^{\prime}}\left\langle\hat{s}_{l d}^{\alpha} \hat{s}_{l^{\prime} d^{\prime}}^{\beta}\right\rangle \int d r^{\prime}\left\langle\delta\left(r+\hat{R}_{l d}-r^{\prime}\right) \delta\left(r^{\prime}-\hat{R}_{l d}(t)\right)\right\rangle
$$

The equation (6) is divided into two parts, the first part describes the correlation of time-dependent between paired spins and the second part is correlation function which describes pair distribution in space-time. Equation (5) consists of contributions of elastic magnetic scattering, elastic scattering in the spin system but inelastic in the phonon system, inelastic magnetic scattering and inelastic scattering in both the spin and phonon systems. In the true paramagnetic state of a localized magnetic system the magnetic ion spins are completely uncorrelated and are randomly oriented. So in this research, contribution of inelastic scattering in both the spin and phonon systems for calculating equation (5) is considered. This situation is perhaps never realized in practice but maybe is true at temperatures much higher than the ordering temperature [12]. For such a system

$\left\langle\widehat{\boldsymbol{S}}_{l}^{\alpha} \widehat{\boldsymbol{S}}_{l^{\prime}}^{\beta}(t)\right\rangle=\delta_{\alpha \beta} \delta_{l^{\prime}, l} \frac{1}{3} \boldsymbol{S}(\boldsymbol{S}+1)$

also $\sum_{\alpha \beta}\left(\delta_{\alpha \beta}-\widehat{\boldsymbol{\kappa}}_{\alpha} \widehat{\boldsymbol{\kappa}}_{\beta}\right)=2$ We have from (4)

$\frac{d^{2} \sigma}{d \Omega d E}=\frac{k^{\prime}}{k} r_{0}^{2}\left(\frac{1}{2} g f(\kappa)\right)^{2} e^{-2 w(\kappa)} \frac{2}{3} s(s+1) \frac{N}{2 \pi \hbar} \times \int_{-\infty}^{\infty} d t e^{-i \omega t}\left(e^{\langle\kappa . u(t) \kappa u(I, t)\rangle}-1\right)$

All measurements are performed with a Mathematica Software version 6 in order to investigate the cross-section changes in term of temperature and phonon wave vector.

\section{RESULTS AND DISCUSSION}

Magnetic fluctuations have been extensively studied for scattering vectors within the 0.6 to $0.8 \mathrm{fm}^{-1}$ interval and temperatures ranging from 0 to $2.5 \mathrm{~T}_{\mathrm{c}}$ where $\mathrm{T}_{\mathrm{c}}$ is critical temperature of $\mathrm{Ce}^{3+}$. Fig. 1 shows typical inelastic neutron cross section of $\mathrm{Ce}^{+3}$ ions for scattering vector at $0.2 \mathrm{~T}_{\mathrm{c}}$ and $T_{c}$ when the scattering vectors are between $0.6-0.8 \mathrm{fm}^{-1}$ which $\kappa$ is considered as k-k'. The maximum and minimum of magnetic cross sections are 3.5 and 0.5 barns in scattering vector $0.6 \mathrm{fm}^{-1}$ and at temperatures of $0.2 \mathrm{~T}_{\mathrm{c}} \mathrm{K}$ and $2.5 \mathrm{~T}_{c}$, respectively. This indicates when scattering vector (energy transfer) increases, the cross-section and then the probability of reaction as inelastic decreases.

Scattering cross sections in interval 0.76-0.80 $\mathrm{fm}^{-1}$ for different temperatures are approximately same. So for scattering vector higher than $0.76 \mathrm{fm}^{-1}$, the scattering cross 
section is independent of temperature. In a known inelastic cross section in different temperatures the outputs overlay various neutron energies.

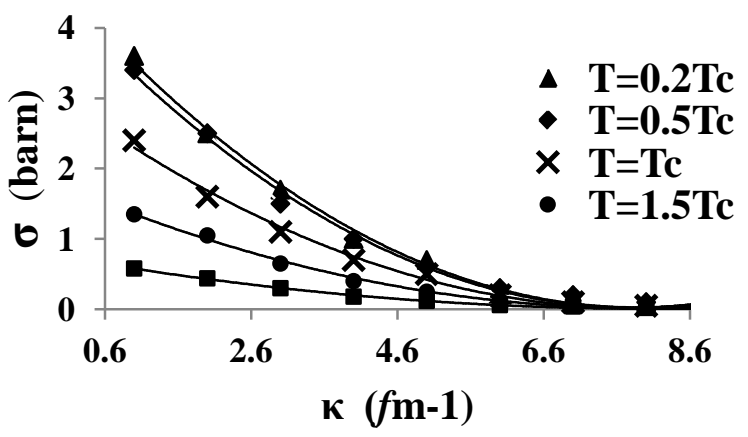

Fig. 1 The total magnetic cross-section in terms of scattering vector in different temperature

The total magnetic cross section changes in terms of temperature for different scattering vectors are shown in Fig.2. For scattering vector $0.6 \mathrm{fm}^{-1}$ when the temperature increases the share of phonon vibrations increases too and the magnetic correlations decreases in $\mathrm{Ce}^{3+}$ lattice. So, the coupling between localized magnetic moments and the itinerant electrons have lower effective role in neutron inelastic scattering. As a result, by increasing scattering vector the phonon vibrations in lattice structure increases which cause an intense drop of inelastic neutron scattering cross section from localized electrons. Fig.2 shows the cross section is independent of temperature in smaller cross sections.

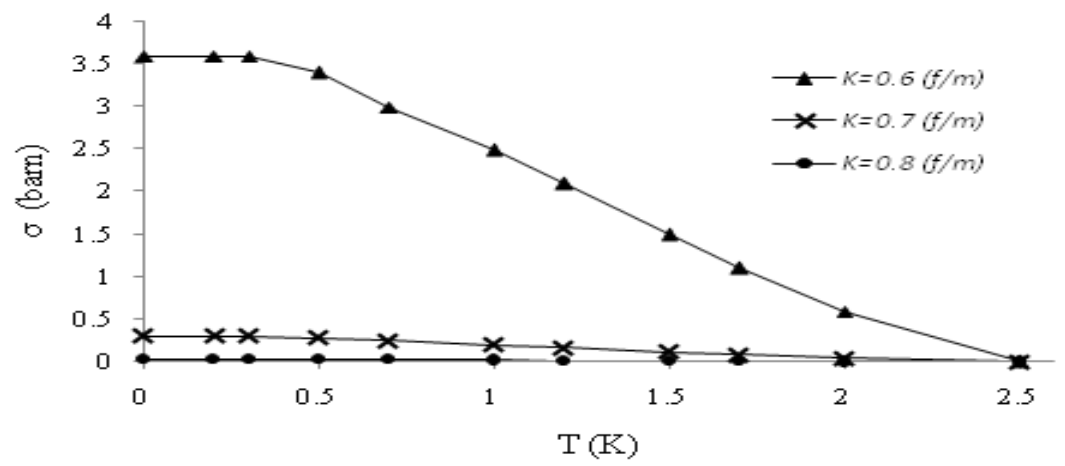

Fig. 2 The temperature dependence of total magnetic scattering cross section for different scattering vectors

By considering electron configuration of $\mathrm{Ce}^{+3}$ in the last layer $\left(4 \mathrm{f}^{1} 5 \mathrm{~s}^{2} \mathrm{p}^{6}\right)$, spin and orbital angular momentum and total angular momentum for $\mathrm{Ce}^{+3}$ are $\mathrm{s}=1 / 2, \mathrm{l}=2, \mathrm{~J}=5 / 2$, respectively. the dependence of total magnetic cross section on phonon wave vector and temperature is shown in Fig.3, $\mathrm{T}$ is considered as reduced temperature $\mathrm{T} / \mathrm{Tc}$. It shows typical inelastic neutron scattering response from $\mathrm{Ce}^{+3}$ ions at $0-2.5 \mathrm{~T}_{\mathrm{c}}$ and the range of phonon wave vector are within $0-0.4 \mathrm{fm}^{-1}$ interval. The maximum obtained cross section is $3 \times 10^{-3}$ barn in temperature $1.2 \mathrm{~K}$, because of the strong bound between localized magnetic moments and the itinerant electrons in $1.2 \mathrm{~K}$ temperatures. The minimum In the Ce sublattice. obtained cross section is 0 barn in temperature $2.5 \mathrm{~T}_{\mathrm{c}}$ indicates the weakly coupled high temperature situation in which the speed of conduction electrons is so much that the coupling makes a mild correlation between the magnetic moment of conduction electrons and the localized electrons. Also, in temperatures higher than Tc the most interaction are nuclear. According to the Fig.3, the increase in temperature results the increase in the phonon vibration contributing so much to total magnetic scattering and the decrease in the spin correlations 


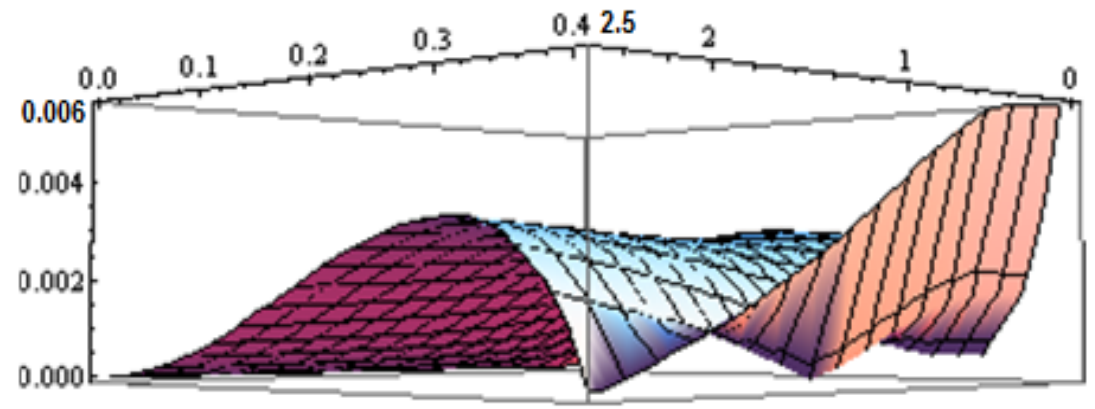

Fig. 3. The changes of magnetic neutron scattering cross-section in terms of temperature and wave vector

It is generally assumed that, in the state with large hybridization between the localized $4 \mathrm{f}$ electrons and the conduction electrons one narrow inelastic peak is made at low temperature about 0.02Tc (Kondo effect) which is shown in Fig.4.(a). This peak with intensity strongly dependent on the wave vector, reflects the coherent origin of the ground state in the cerium lattice. Moreover, coherent peak indicates dynamic magnetic correlation and paramagnetic effective moment in Ce ion. Fig.4.(b) shows the inelastic response of $\mathrm{Ce}^{3+}$ at high temperature $\left(\mathrm{T}_{\mathrm{c}}\right)$ with phonon contribution at phonon wave vector interval 0.4-0.6. Also, two coherent broad peaks is almost $\boldsymbol{\kappa}$-independence and reflect magnetic scattering because of magnetic spin correlation. Moreover, with increasing phonon wave vector interval at higher temperature, the contribution of lattice vibration increases and it causes magnetic uncorreletion.

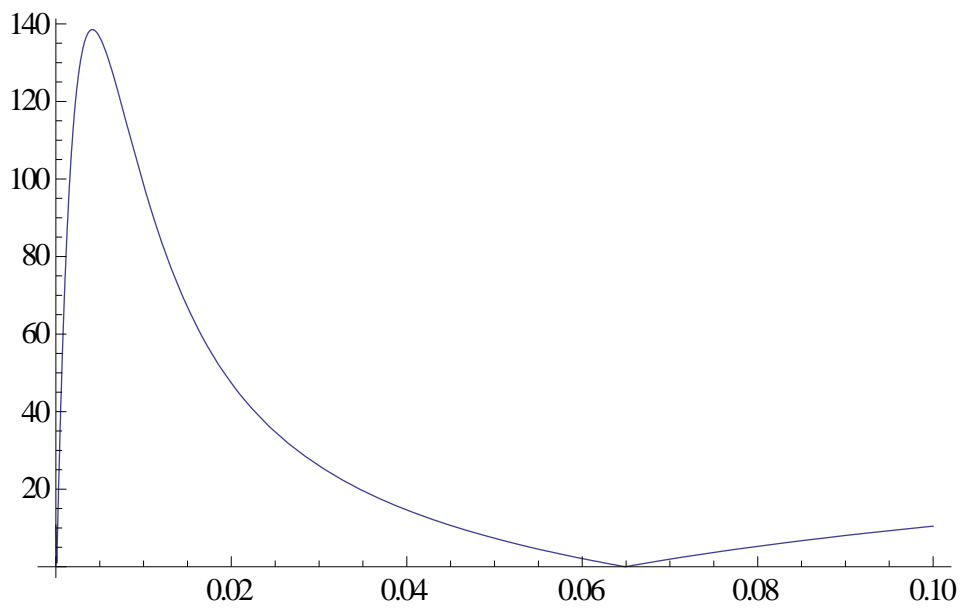

Fig. 4.(a) The total inelastic magnetic scattering cross section in terms of phonon wave vector with a coherent narrow peak at 0.02Tc. 


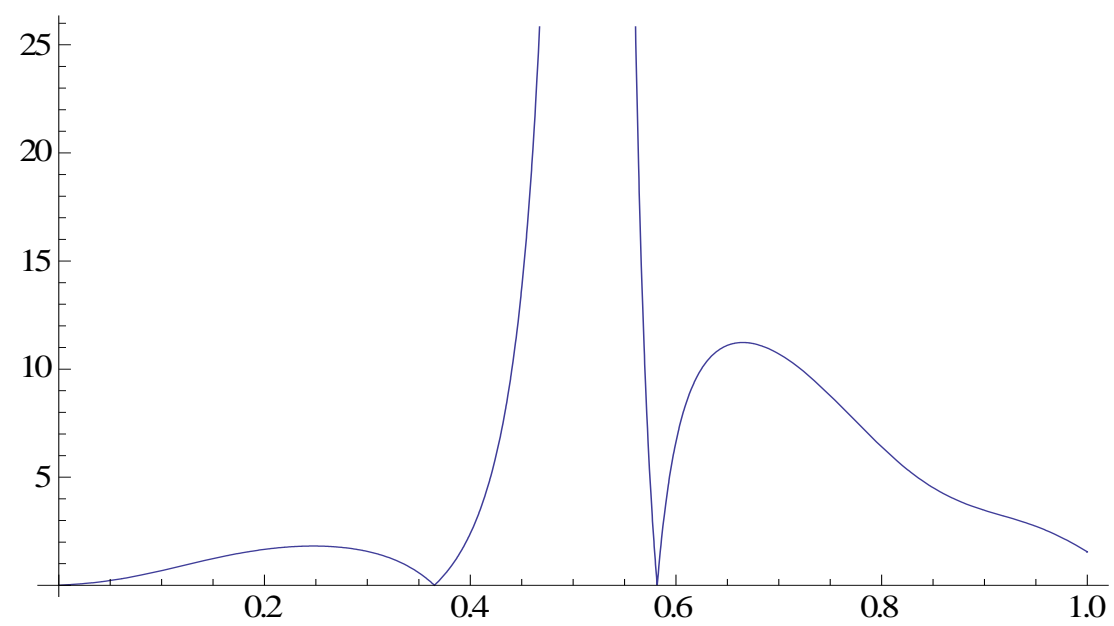

Fig.4.(b).magnetic scattering obtained at larger wave vector interval at Tc.

Supplementary calculation on ferromagnetic crystals shows that the scattering cross section is proportional with temperature and this means that with increasing the distinguish paramagnetic from ferromagnetic crystals.

\section{CONCLUSION}

Investigations of magnetic neutron scattering on $\mathrm{Ce}^{3+}$ crystal could provide valuable information on the magnetic correlation in this system. Dependence total magnetic cross section on phonon and scattering wave vector at low temperature is expressed by Kondo effect and the magnetic scattering intensity is strongly proportional with scattering vector $\boldsymbol{\kappa}$. Generally, total magnetic scattering cross section decreases by increasing temperature. Our results show increasing temperature decreases the reactions probability as an inelastic scattering. The effect of temperature on scattering cross section of an unknown crystal can be used to distinguish different types of crystals.

\section{REFERENCES}

[1] Rossat.Mingnod J, Regnault LP, Jacoud JL, Journal of Magnetism and Magnetic Materials 76\&77(1988) 376-384.

[2] Yamaguchi Y, Thurston TR, Miki H, Tomiyoshi S, Journal of Physica B, 213\&214(1995) 363.

[3] Maekawa S, Kashiba S, Takahashi S, Tachiki M, Journal of Magnetism and Magnetic Materials 52(1985) 149-151.

[4] Lovesey S,Vol. 2, Oxford Science Publication 1 (1987).

[5] Marshall W, Lovesey SW, Oxford University Press (1971).

[6] Squires GL, Cambridge University Press (1978).

[7] Lovesey S, Vol. 1, Oxford Science Publications (1987).

[8] Price D L, Sköld K, , Vol. 23, Academic Press,London (1987).

[9] Izyumov Yu A, Ozerov RP,Plenum Press, New York ( 1970).

[10] Chatterji T, France chapter 1. 15 (2006).

[11] Brown P J, Vol. C, eds. A. J. C. Wilson and E. Prince, Second Edition, p. 450, Kluwer Academic Publishers, Dordrecht, (1999).

[12] Colognesi D, Journal of Physica B, 89 (2007) 398. 